\title{
Analysis of different vibration patterns to guide blind people
}

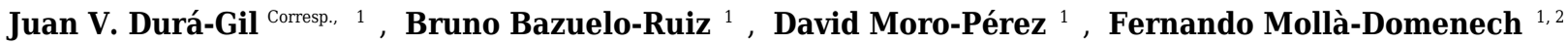 \\ 1 Instituto de Biomecánica de Valencia, Universitat Politècnica de València, Valencia, Spain \\ 2 Healthcare Technology Group, CIBER's Bioengineering, Biomaterials and Nanomedicine (CIBER-BBN), Valencia, Spain \\ Corresponding Author: Juan V. Durá-Gil \\ Email address: juan.dura@ibv.upv.es
}

The literature indicates the best vibration positions and frequencies on the human body where tactile information is transmitted. However, there is a lack of knowledge about how to combine tactile stimuli for navigation. The aim of this study is to compare different vibration patterns outputted to blind people and to determine the most intuitive vibration patterns to indicate direction for navigation purposes through a tactile belt. The vibration patterns that stimulate the front side of the waist are preferred for indicating direction. Vibration patterns applied on the back side of the waist could be suitable for sending messages such as stop. 
Title: Analysis of different vibration patterns to guide blind people

Juan V. Durá-Gil ${ }^{1}$, Bruno Bazuelo-ruiz ${ }^{1}$, David Moro-Pérez ${ }^{1}$, Fernando MollàDomenech $^{1,2}$.

(1) Instituto de Biomecánica de Valencia, Universitat Politécnica de València, València, Spain.

(2) Healthcare Technology Group, CIBER's Bioengineering, Biomaterials and Nanomedicine (CIBER-BBN)

Corresponding author:

Juan Vicente Durá-Gil

juan.dura@ibv.upv.es 


\section{Abstract}

2 The literature indicates the best vibration positions and frequencies on the human body

3 where tactile information is transmitted. However, there is a lack of knowledge about how

4 to combine tactile stimuli for navigation. The aim of this study is to compare different

5 vibration patterns outputted to blind people and to determine the most intuitive vibration

6 patterns to indicate direction for navigation purposes through a tactile belt. The vibration

7 patterns that stimulate the front side of the waist are preferred for indicating direction.

8 Vibration patterns applied on the back side of the waist could be suitable for sending

9 messages such as stop.

\section{Introduction}

Visual sensory substitution can take a variety of forms and may be mediated by a device, such as a global positioning system (GPS), a cell phone (Ranjbar \& Stenström, 2013) or a PDA (Ghiani, Leporini \& Paternò, 2009). However, their use is limited to familiar

14 environments (Kärcher et al., 2012) or museums (Ghiani, Leporini \& Paternò, 2009).

15 Faugloire and Lejeune (2014) compared spatial language with tactile guidance and obtained better responses with vibrations. Other relevant aspects are the number of 17 vibrating elements, known as tactors, 6 to 64, (Cholewiak \& Craig, 1984; Cholewiak, Brill \& Schwab, 2004; Faugloire \& Lejeune, 2014) and the body site where the vibration is applied. Typical body sites include the thigh (Cholewiak \& Craig, 1984), finger (Cholewiak, 1999; Ghiani, Leporini \& Paternò, 2009), palm (Cholewiak \& Craig, 1984), back

21 (Srikulwong \& O'Neill, 2010) and waist (Faugloire \& Lejeune, 2014). A disadvantage of

22 the devices worn on the hand or fingers is that they limit the freedom to take or manipulate 
23 something with the hand itself. Placing tactors on the waist frees up the upper limbs to 24 perform any act freely. Due to the torso being relatively flat, stable, large and easily 25 accessible compared to the limbs, it is a good option for indicating direction (Johnson \& 26 Higgins, 2006).

Although the amount of information that can be perceived through touch is less than that which can be perceived through vision (Ghiani, Leporini \& Paternò, 2009), haptic devices have the advantage that they can be useful in noisy environments (Marston et al., 2006).

However, there is a lack of information in the literature about what vibration patterns are more intuitive for navigation purposes. This paper presents a preliminary study regarding the feasibility of guiding blind people with a belt that applies tactile stimuli. This study is part of a project that aims to develop a running facility embedded in a $400 \mathrm{~m}$ athletic track for visually impaired people to run independently without the assistance of others. If tactile guidance is feasible, the project expects to develop real-time tracking of blind runners based on radio-frequency position detection technology (RFID), which can be deployed in a stadium around a running track and is able to operate in real-time. A belt is considered a feasible solution for runners because it will not disrupt their movements. The aim of the present study is to compare different vibration patterns in order to define design criteria for guiding blind people by means of tactile navigation devices. Moreover, considering that recruitment of an adequate number of blind people might be difficult in 43 future project phases, this preliminary study analyses the feasibility of undertaking tests 44 with blindfolded participants that have no sight impairments. This data will allow us to 45 determine if the vibration patterns designed are able to provide tactile information so as 
46 to continuously specify the intended direction relative to the current destination of the 47 user.

48 Methods

49

50

51

52

53

54

55

56

57

58

59

60

61

62

63

64

65

66

67

\section{Participants}

Twenty people participated in a study that included three experiments. The main criterion used to select blind people was that they needed to use a long cane to find their way and avoid obstacles. Sighted people were blindfolded during the experiments. None of the participants had previously worn or had any experience with vibrotactile displays.

In the first and second experiments, there were a total of twelve subjects: 6 blind ( 3 women and 3 men) and 6 sighted people ( 3 women and 3 men); age: $32.17 \pm 9.92$ years; mass: $65.75 \pm 10.73 \mathrm{~kg}$; height: $1.71 \pm 0.05 \mathrm{~m}$; BMI: 22.4 \pm 3.1 ; waist circumference: $82.2 \pm 10.3 \mathrm{~cm}$.

Participants did the first and second experiment consecutively on the same day under laboratory conditions (indoors).

The third experiment was performed one month later (outdoor). Different participants were recruited because the previous participants were not available. Eight people took part in the study: 4 blind ( 3 man and 1 woman) and 4 sighted people ( 2 women and 2 men); age: $33.57 \pm 10.11$ years; mass: $65.57 \pm 10.40 \mathrm{~kg}$; height: $1.69 \pm 0.05$ m; BMI: 22.81 \pm 2.81 ; waist circumference: $79.66 \pm 4.03 \mathrm{~cm}$.

Each participant volunteered and the information and informed consent was provided and signed by all subjects. The study protocol received approval by the Ethics

7 Committee (Universitat Politècnica València): approval number: 765 - 16/07/2013. 
68

69

70

711 was placed $25 \mathrm{~mm}$ over the navel. Tactor 5 was placed on the spine. The other tactors

72 were equally spaced between tactor 1 and tactor 5 (Figure 1). We selected this placement

73 for the belt because it has been used in the literature to transmit tactile information

74 successfully (Cholewiak, Brill \& Schwab, 2004). The waist circumference was measured

75 at this level. Tactors were equally distributed. The stimuli transmitted wirelessly consisted 76

77 in the technical specification data sheet of the "Science Suit" provided by Elitac:

78

79

80

81 Subjects chose the vibration intensity according to their preference. The vibrotactile signal 82 was perceived well by all participants.

- Vibration frequency of $158.3 \pm 2.4 \mathrm{~Hz}$.

- Maximum vibration strength: $55.5 \pm 9.5 \mathrm{~m} / \mathrm{s}^{2}$.

- Tactor outer dimensions (I $\times$ w x h): 34x16x11 mm. 


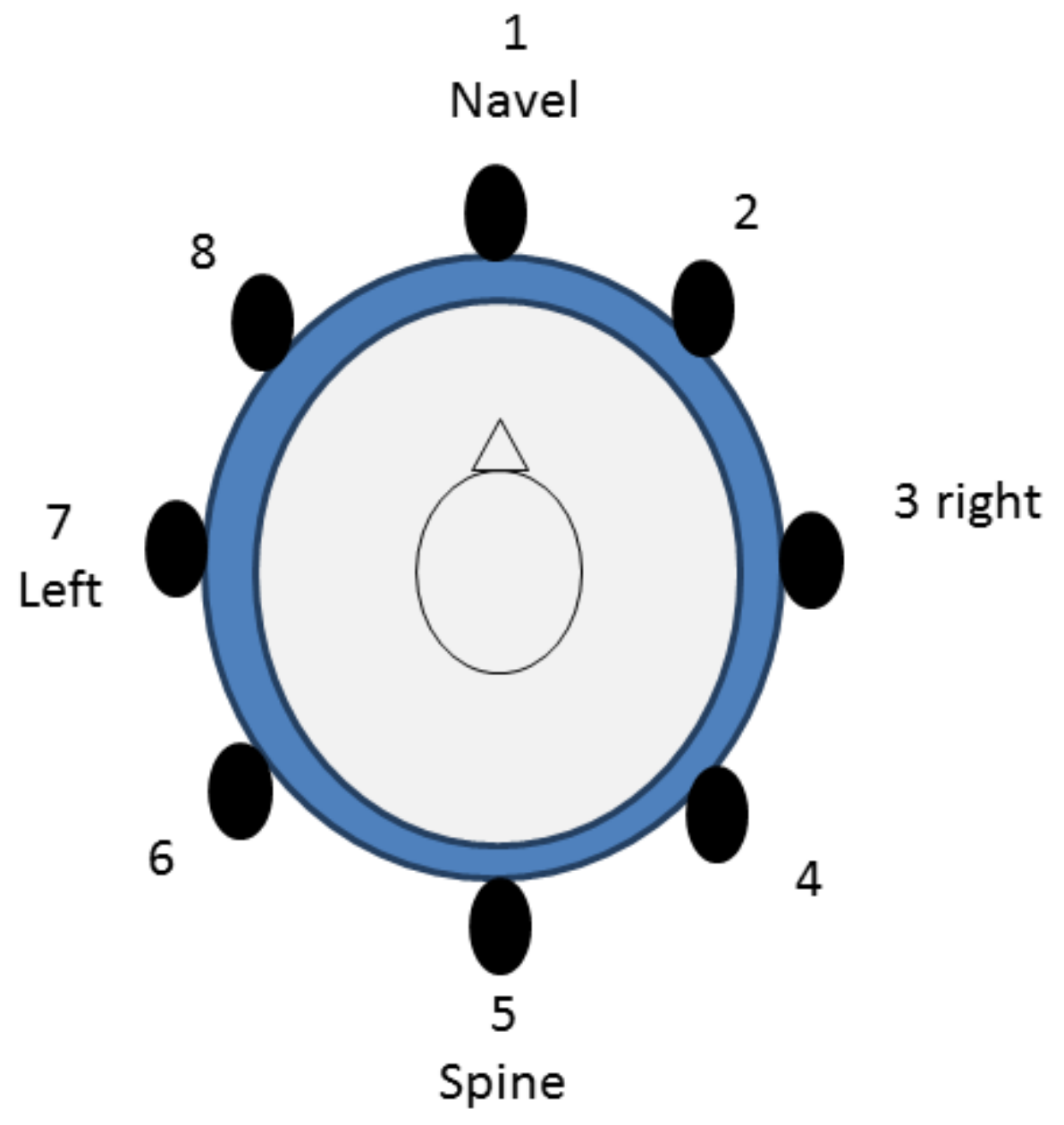

Figure 1: Position of the tactors (top view).

In the first experiment, 13 vibration patterns were outputted and the subjects did a multiple choice questionnaire: left, right, go ahead, other, or not answer. The subject

87 provided an oral description if "other" was selected (i.e. go back, stop, etc.). The patterns were chosen in order to analyse differences between: (i) stimuli applied in the anterior region vs. dorsal region, and (ii) stimuli applied in sequences vs. fixed area. The vibration patterns presented are the following: A01: tactor 1 (navel); A02: tactor 5 (spine); A03:

91 tactors 3 and 7 at the same time; A04: tactor 7 (left); A05: tactor 3 (right); A06: sequence 2-3-4; A07: sequence 8-7-6; A08: sequence 4-3-2; A09: sequence 6-7-8; A10: sequence 
The tactors of patterns A01 to A05 (no sequence) were $200 \mathrm{~ms}$ active and $500 \mathrm{~ms}$

95

96

97

98

99

100

101

102

103

104

105

106

107

108

109

110

111

112

113

114

115

116

off. Patterns A06 to A13 were a sequence repeated a number of times, with each tactor active $200 \mathrm{~ms}$. The subjects were prevented from hearing the vibratory stimuli. The vibration patterns were outputted to the participants in a randomized order.

For each subject in the second experiment, the previous results were classified by the responses given (left, right, continue ahead, other and no answer). The aim of this phase was to compare the vibration in pairs. The subjects do pairwise comparisons, through the Analytic Hierarchy Process (Saaty, 1990). The subject selects the best pattern to indicate "turn left" or "turn right".

The first and second experiments were designed to choose the most suitable vibration patterns for future experiments and to confirm that tests with blindfolded sighted people were feasible.

The third experiment was performed outdoors on a real scale $(1: 10$, length $40 \mathrm{~m}$, $0.90 \mathrm{~m}$. width) athletic track. Three vibration patterns (Table 1) were outputted and the subjects had to perform two laps walking for each pattern to determine whether a learning process occurs between the first and the second lap. The vibration patterns were outputted to the participants in a randomized order. Blind people can feel insecure if there is no tactile feedback. For this reason, if the participant walks in the right direction, tactor 1 (navel) is always active. The vibration patterns were designed on the basis of the results obtained in the previous experiments. The belt was connected to a laptop by Bluetooth. A researcher controlled the pattern transmitted using the laptop. During the laps, the number of times that the subject leaves the track was recorded. After performing the two laps, the researcher asked the participant their thoughts on the pattern. The scores given 
117 by participants are the followings: 1 - feel lost or not guided; 2- guided badly; 3- guided

118 normally; 4- guided well; 5- very confident or very well guided.

119 Table 1: Characteristics of the vibration patterns. $\theta$ is the angle the subject should turn.

120 On is the time that the tactor is active. Off is the time that the tactor is not active.

\begin{tabular}{|c|c|c|c|}
\hline & VP1 & VP2 & VP3 \\
\hline $\begin{array}{l}\text { Continue } \\
\text { straight }\end{array}$ & $\begin{aligned} \text { Tactor } 1 \\
\\
\text { On }=200 \mathrm{~ms} \\
\text { Off }=500 \mathrm{~ms}\end{aligned}$ & $\begin{aligned} \text { Tactor } 1 \\
\text { On }=200 \mathrm{~ms} \\
\text { Off }=500 \mathrm{~ms}\end{aligned}$ & $\begin{array}{r}\text { Tactor } 1 \\
\text { On }=200 \mathrm{~ms} \\
\text { Off }=500 \mathrm{~ms}\end{array}$ \\
\hline Turn right & $\begin{array}{l}\text { If } \theta<45^{\circ} \text {, then tactor } 2 \text { is } \\
\text { active. If } \theta \geq 45^{\circ} \text {, then } \\
\text { tactor } 3 \text { is active } \\
\quad \text { On }=200 \mathrm{~ms} \\
\quad \text { Off }=500 \mathrm{~ms}\end{array}$ & $\begin{array}{l}\text { Time Off decreases with } \\
\text { angle }(\theta) \text {. If } \theta<45^{\circ} \text {, then } \\
\text { tactor } 2 \text { is active. If } 45^{\circ} \leq \theta \\
<90^{\circ} \text {, then tactor } 3 \text { is } \\
\text { active. } \\
\qquad \text { On }=100 \mathrm{~ms} \\
\text { off }=500\left(1-\frac{\theta}{90}\right) \mathrm{ms} \\
\text { If } \theta \geq 90^{\circ} \text {, then tactor } 3 \text { is } \\
\text { always active, and: } \\
\text { Off }=0 \mathrm{~ms}\end{array}$ & $\begin{array}{l}\text { Sequence of tactors 1-2-3. } \\
\text { If } \theta<90^{\circ} \text {, then } \\
\quad O n=100 \mathrm{~ms} \\
\quad O f f=500\left(1-\frac{\theta}{90}\right) \mathrm{ms} \\
\text { If } \theta \geq 90^{\circ} \text {, then: } \\
\text { Off }=0 \mathrm{~ms}\end{array}$ \\
\hline Turn left & $\begin{array}{l}\text { The same as right, but with } \\
\text { tactors } 8 \text { and } 7\end{array}$ & $\begin{array}{l}\text { The same as right, but } \\
\text { with tactors } 8 \text { and } 7 .\end{array}$ & $\begin{array}{l}\text { The same as right, but } \\
\text { sequence of tactors } 1-8-7 \text {. }\end{array}$ \\
\hline
\end{tabular}

121

122

123

124

125

126

127

128

129

130

131

\section{Statistical analyses}

In the first experiment, we analysed the differences between blind and sighted people with a Fisher's exact test because the sample size is small. Moreover, in experiment 1 and 3, we performed a frequency analysis with an additional qualitative assessment of the opinion and feelings of all participants.

Patterns were obtained from the first experiment to indicate direction. Several patterns may be feasible to indicate the same direction. In experiment 2, the Analytic Hierarchy Process (Saaty, 1990) was carried out in order to get the most suitable vibration pattern of the same category (left, right or continue ahead). The subjects did pairwise comparisons. The order of presentation is randomized. The subjects compared patterns 
132 in pairs to judge which one is preferred for each direction. For example: A01 is better than

133 A02 to indicate turn left. This methodology transforms the pairwise comparisons into a

134 score of 0 to 100 for each vibration pattern. The score is used as an independent variable.

135 Thereafter, a one-way analysis of means and a pairwise comparison with the Bonferroni 136 method were performed.

137 Two-way analysis of variance (ANOVA) was used to examine the influence of the 138 vibration pattern and lap order on the number of times that the subject leaves the track.

139 Statistical power analysis is performed for the three experiments separately. We 140 use a contingency table in experiment 1 . Therefore, we used the approach for Chi-square 141 tests: Cohen's w (effect size). We use ANOVA in experiments 2 and 3, so therefore we 142 apply Cohen's f for measuring the effect size (Cohen, 1988).

\section{Results}

Table 2 shows the frequencies of the vibration patterns selected for each category by the participants in the first experiment. A score of $\geq 10$ is considered a valid vibration pattern for this category. There are no significant differences between blind and sighted

147 people for any of the patterns and in any of the categories (Fisher's exact test, $p$-value > 148 0.05). However, the power of test was found to be 0.3 . Cohen suggests that effect size 149 index values of 0.5 represents a large effect size. Power with a value of 0.8 would need 15038 observations instead of 12. 
152 Table 2: Frequencies and percentages of the vibration patterns selected by the subjects

153 in the first experiment. R: right; L: left; C: continue; O: other; NA's: no answer.

154 Percentages higher than $80 \%$ are highlighted.

\begin{tabular}{|c|c|c|c|c|c|c|c|c|c|c|c|c|c|}
\hline & A01 & A02 & A03 & A04 & A05 & A06 & A07 & A08 & A09 & A10 & A11 & A12 & A13 \\
\hline \multirow{2}{*}{$\mathrm{R}$} & 0 & 0 & 0 & 1 & 11 & 10 & 2 & 5 & 6 & 11 & 1 & 4 & 4 \\
\hline & $0 \%$ & $0 \%$ & $0 \%$ & $8 \%$ & $92 \%$ & $83 \%$ & $17 \%$ & $42 \%$ & $50 \%$ & $92 \%$ & $8 \%$ & $33 \%$ & $33 \%$ \\
\hline \multirow{2}{*}{ L } & 0 & 0 & 0 & 11 & 1 & 2 & 10 & 5 & 6 & 1 & 10 & 5 & 4 \\
\hline & $0 \%$ & $0 \%$ & $0 \%$ & $92 \%$ & $8 \%$ & $17 \%$ & $83 \%$ & $42 \%$ & $50 \%$ & $8 \%$ & $83 \%$ & $42 \%$ & $33 \%$ \\
\hline \multirow{2}{*}{ C } & 11 & 2 & 5 & 0 & 0 & 0 & 0 & 0 & 0 & 0 & 1 & 2 & 2 \\
\hline & $92 \%$ & $17 \%$ & $42 \%$ & $0 \%$ & $0 \%$ & $0 \%$ & $0 \%$ & $0 \%$ & $0 \%$ & $0 \%$ & $8 \%$ & $17 \%$ & $17 \%$ \\
\hline \multirow{2}{*}{0} & 1 & 10 & 7 & 0 & 0 & 0 & 0 & 0 & 0 & 0 & 0 & 0 & 0 \\
\hline & $8 \%$ & $83 \%$ & $58 \%$ & $0 \%$ & $0 \%$ & $0 \%$ & $0 \%$ & $0 \%$ & $0 \%$ & $0 \%$ & $0 \%$ & $0 \%$ & $0 \%$ \\
\hline \multirow{2}{*}{ NA's } & 0 & 0 & 0 & 0 & 0 & 0 & 0 & 2 & 0 & 0 & 0 & 1 & 2 \\
\hline & $0 \%$ & $0 \%$ & $0 \%$ & $0 \%$ & $0 \%$ & $0 \%$ & $0 \%$ & $17 \%$ & $0 \%$ & $0 \%$ & $0 \%$ & $8 \%$ & $17 \%$ \\
\hline \multirow{2}{*}{ Total } & 12 & 12 & 12 & 12 & 12 & 12 & 12 & 12 & 12 & 12 & 12 & 12 & 12 \\
\hline & $100 \%$ & $100 \%$ & $100 \%$ & $100 \%$ & $100 \%$ & $100 \%$ & $100 \%$ & $100 \%$ & $100 \%$ & $100 \%$ & $100 \%$ & $100 \%$ & $100 \%$ \\
\hline
\end{tabular}

155

156

In Table 2, for the category "Right", vibration patterns 5, 6 and 10 were the most

157 preferred. For category "Left", vibration patterns 4, 7 and 11, and to continue ahead

158 vibration pattern 1 were the most suitable for the majority of participants. Vibration pattern

1592 was chosen by 10 of a total of 12 subjects as a vibration pattern that suggests to them

160 other information. They indicated to us that the vibration pattern 2 means to stop or turn

$161180^{\circ}$.

162 For "Continue", A01 is the most suitable vibration pattern for the majority of

163 subjects (Table 2). However, several patterns are suitable to indicate "turn left" and "turn

164 right". Therefore, we compared these patterns in experiment 2. For "Right" (Figure 2-A), 
165 the vibration patterns compared are A10, A06 and A05 and the results reveal that there 166 are significant differences (t-test $\mathrm{p}<0.05$ ) between A05 and A06 and also A05 and A10.

167 However, no significant difference was found between A10 and A06. For "Left" (Figure 2168 B), the vibration patterns compared were A04, A07 and A11 and the results show 169 statistical significance (t-test $p<0.05$ ) between A04 and A07 and A04 and A11, but not 170 between $\mathrm{A} 07$ and $\mathrm{A} 11$. Table 3 shows the mean and standard deviation for the vibration 171 patterns compared. The power of the test is 0.8 for the left direction and 0.9 for the right 172 direction.

A

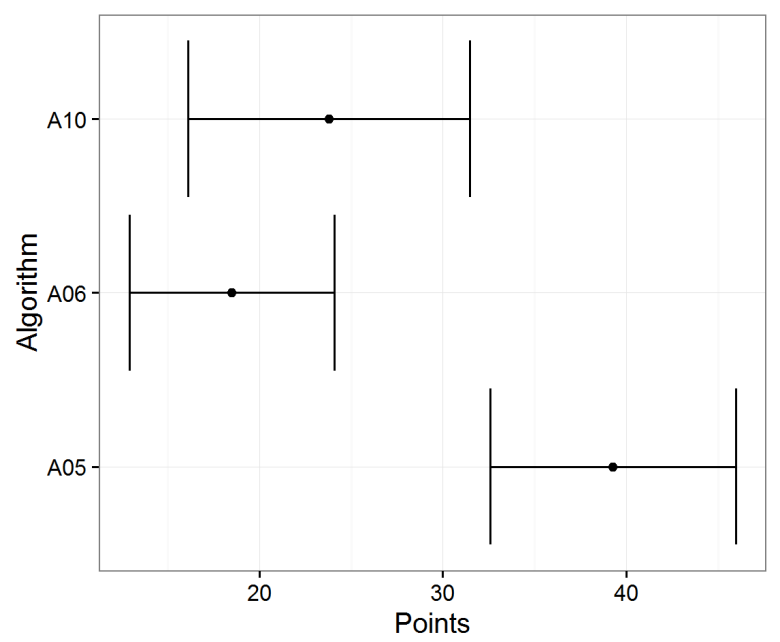

B

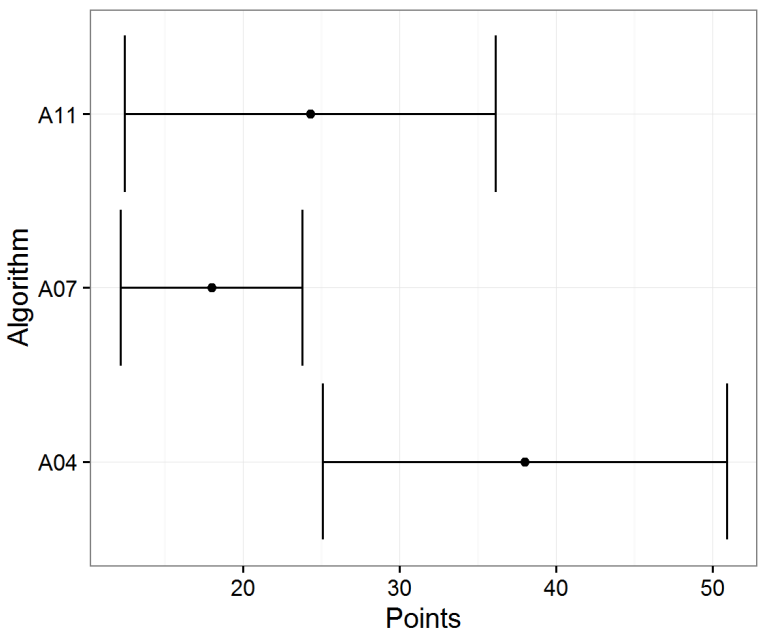

173

Figure 2: Scores given by the Analytic Hierarchy Process (Saaty, 1990) for each

175 vibration pattern. (A) Right direction. (B) Left direction.

176 Table 3: Mean and St. Deviation of the scores.

\begin{tabular}{|l|l|r|r|}
\hline Direction & \multicolumn{1}{|c|}{ Pattern } & \multicolumn{1}{c|}{ Mean } & $\begin{array}{c}\text { St. } \\
\text { Deviation }\end{array}$ \\
\hline Left & A04 & 38 & 12.89 \\
\hline Left & A07 & 18 & 5.81 \\
\hline Left & A11 & 24.3 & 11.84 \\
\hline Right & A05 & 39.27 & 6.69 \\
\hline Right & A06 & 18.5 & 5.58 \\
\hline Right & A10 & 23.8 & 7.67 \\
\hline
\end{tabular}


In experiment 3 , we detected that in the second lap the number of times that most

179 subjects leave the track tends to decrease: the mean of the differences was -0.49 (one180 sided paired t-test, $p<0.05)$.

Two-way analysis of variance (ANOVA) shows no significant differences between

182 the vibration patterns and participant condition (sighted or blind) ( $p>0.05$ ), neither for 183 total errors nor errors by lap (Table 4). The power of the test is 0.5 . Considering an effect 184 size of 0.5 , a power of 0.8 would need fourteen subjects instead of eight.

185 Table 4: Number of times that subjects left the track. VP: vibration pattern.

\begin{tabular}{|l|c|c|c|c|c|c|}
\hline & \multicolumn{2}{|c|}{ VP 1 } & \multicolumn{2}{c|}{ VP 2 } & \multicolumn{2}{c|}{ VP 3 } \\
\cline { 2 - 7 } & Lap 1 & Lap 2 & Lap 1 & Lap 2 & Lap 1 & Lap 2 \\
\hline Subject 1 & 3 & 3 & 6 & 4 & 4 & 2 \\
\hline Subject 2 & 4 & 3 & 5 & 5 & 6 & 5 \\
\hline Subject 3 & 2 & 0 & 0 & 0 & 3 & 0 \\
\hline Subject 4 & 0 & 2 & 5 & 2 & 1 & 1 \\
\hline Subject 5 & 1 & 1 & 4 & 3 & 3 & 4 \\
\hline Subject 6 & 2 & 2 & 2 & 0 & 3 & 2 \\
\hline Subject 7 & 3 & 2 & 2 & 1 & 1 & 0 \\
\hline Subject 8 & 4 & 4 & 3 & 2 & 2 & 0 \\
\hline
\end{tabular}

186

187 Considering the opinion of the participants (Table 5), there are not any significant 188 differences between patterns (Fisher's exact test, p-value > 0.05). However, the opinions and comments of the participants indicate a higher self-confidence with the vibration patterns presented by the vibrotactile device. No participant considered that VP1 and VP2

191 provided bad guidance or made them feel lost. Only two participants considered that VP3 192 provided bad guidance or made them feel lost. 
194 Table 5: Opinion of the participants.

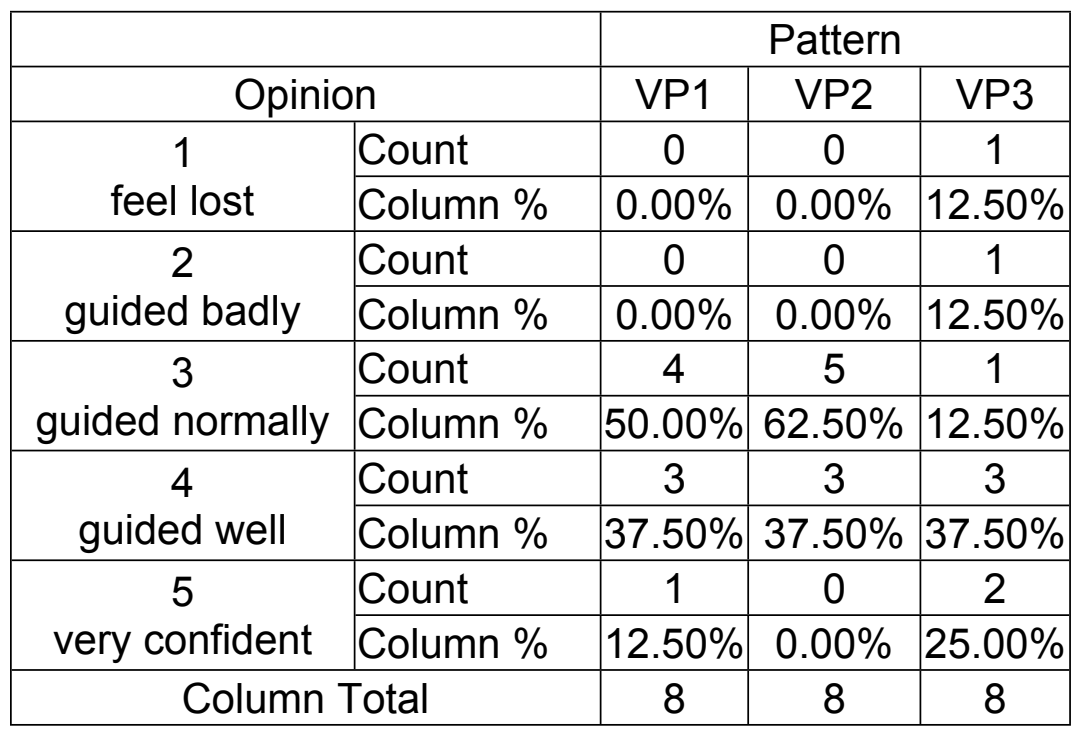

195

\section{Discussion}

The aims of this study were to identify what the different vibration patterns 198 outputted suggest to blind and sighted people and to determine the most intuitive vibration pattern to indicate direction through a tactile belt. Furthermore, this data could provide us with real insight into the effectiveness of a novel guiding system in a dynamic situation.

201 Other researchers (Cholewiak, Brill \& Schwab, 2004; Faugloire \& Lejeune, 2014) evaluate

202 the effectiveness of the tactile stimulus with fixed directions or fixed angles (e.g. $45^{\circ}, 90^{\circ}$, $203135^{\circ}, 180^{\circ}$, etc.). Instead, we use a dynamic feedback approach that tells the subject to 204 rotate more or less. To the best of our knowledge, there is no other study that investigates 205 the response to different vibration patterns outputted on the back and waist by a tactile 206 device in a static and dynamic situation using blind people as subjects

207 Regarding differences between blind and sighted people, the results are not conclusive 208 and more research is needed with an increased number of subjects. 
Regarding the vibration rhythm, Faugloire and Lejeune (2014) applied two 210 vibration rhythms: long ( 1 s ON/ 4 s OFF vibrations) before movement and short (200 ms 211 ON/ 200 ms OFF vibrations) during body rotation. They found greater accuracy with the 212 short tactile mode. Similarly, one stimulus (van Erp, Carter \& Andrew, 2006; van Erp, 213 2008) was activated in a $100 \mathrm{~ms}$ ON/ $200 \mathrm{~ms}$ pattern. We used the time of activation of 214 these studies but we increased the gap between bursts so the user would feel an 215 intermittent rhythm rather than a continuous vibration. Therefore, our vibration rhythm 216 was $200 \mathrm{~ms}$ or $100 \mathrm{~ms}$ ON depending on the degree of turning and $500 \mathrm{~ms}$ OFF. We aim 217 to ensure a rapid response by participants in order to correct their orientation at each 218 moment. Our results indicate that single bursts are better to indicate direction through a 219 tactile belt.

220 Faugloire and Lejeune (2014) used a belt with the location of tactile stimulation 221 continuously indicating the requested direction relative to the current orientation of the 222 participants. The location of tactile stimulation is updated along with the body rotation of 223 the user. The authors used all tactors to indicate direction. In contrast, our system also 224 used 8 tactors but only those of the anterior part of the waist are employed to indicate 225 direction. The tactors of the posterior part of the waist may be used to indicate other 226 messages like stop or reduce speed. This is a clear and simple way of offering guidance 227 in a practical application.

228 Finally, we find considerable individual variability in the results of the third 229 experiment. Possible causes of variability are the vibration patterns order (training effect), 230 and levels of motivation and concentration. 
231 A limitation of this study is the sample size. Caution must be applied, as the findings

232 might not be applicable to the wider population. Further data collection is required to

233 determine exactly the effectiveness of the different patterns employed.

\section{Conclusions}

235 The present findings will be of great value in the design of tactile devices to guide 236 blind people in physical activities like jogging or running. This study indicates that a belt 237 with tactile stimuli is a feasible solution for guiding blind runners on athletic tracks. The 238 results indicate that single bursts might be better than sequence vibration patterns. 239 However, this result should be confirmed in further research involving more participants. 240 The guiding process could be improved with adequate training.

241 The information provided by the belt led to a positive emotional impact on 242 participants with enhanced feelings of security. The stimuli might be optimised in terms 243 of number of stimulators, vibration rhythm and frequency, and body locus of stimulation.

$244 \quad$ Further work is necessary to test the vibration patterns in real conditions including 245 running on a real athletic track. Running might provoke more impacts and stronger 246 movements than walking. A new belt design might be necessary to prevent vibration and 247 movements while running.

\section{References}

249 Cholewiak RW. 1999. The perception of tactile distance: influences of body site, space, 250 and time. Perception 28:851-875. 
251 Cholewiak R., Brill J., Schwab A. 2004. Vibrotactile localization on the abdomen: Effects 252 of place and space. Attention, Perception, \& Psychophysics 66:970-987. DOI: 10.3758/BF03194989.

254 Cholewiak RW., Craig JC. 1984. Vibrotactile pattern recognition and discrimination at 255 several body sites. Perception \& Psychophysics 35:503-514. DOI: 10.3758/BF03205946.

257 Cohen J. 1988. Statistical power analysis for the behavioral sciences. Hillsdale, N.J: L. $258 \quad$ Erlbaum Associates.

259 van Erp J. 2008. Absolute localization of vibrotactile stimuli on the torso. Attention, 260 Perception, \& Psychophysics 70:1016-1023. DOI: 10.3758/PP.70.6.1016.

261

van Erp J., Carter J., Andrew I. 2006. Iso's work on tactile and haptic interaction 262 guidelines. In: Proceedings of Eurohaptics. Paris, 467-470.

263 Faugloire E., Lejeune L. 2014. Evaluation of heading performance with vibrotactile 264 guidance: the benefits of information-movement coupling compared with spatial 265

266 language. Journal of Experimental Psychology. Applied 20:397-410. DOI: 10.1037/xap0000032.

Ghiani G., Leporini B., Paternò F. 2009. Vibrotactile feedback to aid blind users of mobile 268 guides. Journal of Visual Languages \& Computing 20:305-317. DOI: 269 10.1016/j.jvlc.2009.07.004.

270 Johnson LA., Higgins CM. 2006. A Navigation Aid for the Blind Using Tactile-Visual 271 Sensory Substitution. In: 28th Annual International Conference of the IEEE 272 Engineering in Medicine and Biology Society, 2006. EMBS '06. 6289-6292. DOI: 10.1109/IEMBS.2006.259473. 
274 Kärcher SM., Fenzlaff S., Hartmann D., Nagel SK., König P. 2012. Sensory augmentation 275 for the blind. Frontiers in Human Neuroscience 6:37. DOI: $276 \quad 10.3389 /$ fnhum.2012.00037.

277 Marston JR., Loomis JM., Klatzky RL., Golledge RG., Smith EL. 2006. Evaluation of 278 Spatial Displays for Navigation Without Sight. ACM Trans. Appl. Percept. 3:110279 124. DOI: $10.1145 / 1141897.1141900$.

280 Ranjbar P., Stenström I. 2013. Monitor, a vibrotactile aid for environmental perception: a 281 282 field evaluation by four people with severe hearing and vision impairment. TheScientificWorld Journal 2013:206734. DOI: 10.1155/2013/206734.

283 Saaty TL. 1990. Desicion making by the analytic hierarchy process: Theory and 284 applicationsHow to make a decision: The analytic hierarchy process. European 285 Journal of Operational Research 48:9-26. DOI: 10.1016/0377-2217(90)90057-I.

286 Srikulwong M., O’Neill E. 2010. A Comparison of Two Wearable Tactile Interfaces with a 287 Complementary Display in Two Orientations. In: Nordahl R, Serafin S, Fontana F, Brewster S eds. Haptic and Audio Interaction Design. Lecture Notes in Computer

289 Science. Springer Berlin Heidelberg, 139-148. DOI: 10.1007/978-3-642-15841290 4_15.

291 